\title{
Analysis on Clinical Effects of Humanistic Nursing Care on Chronic Pelvic Inflammatory Disease
}

\author{
Q. SHEN* AND LILING TANG ${ }^{1}$ \\ Gynaecology Department, Shaoxing City Keqiao District Hospital of Traditional Chinese Medicine, Shaoxing 312030, ${ }^{1}$ The De- \\ partment of Obstetrics and Gynecology, the Affiliated Huai'an Hospital of Xuzhou Medical University and the Second People's \\ Hospital of Huai'an, Huai'an 223002, China
}

\section{Shen et al.: Clinical Effects of Humanistic Nursing Care on Chronic Pelvic Inflammatory Disease}

\begin{abstract}
To analyze clinical effects of humanistic nursing care model on patients with chronic pelvic inflammatory disease. Methods apply humanistic nursing care for patients with chronic pelvic inflammatory disease, including psychological care, humane care, dietary intervention, discharge guidance and other comprehensive humanistic nursing care measures, analyze and record patients, changes in anxiety and depression after implementation of nursing care, assess efficacy of clinical care. Patient's anxiety, depression is significantly relieved after implementation of humanistic nursing care for patients with chronic pelvic inflammatory disease. Effective rate of clinical care is relatively high. Humanistic nursing care model is an effective model of care, which can significantly relieve patient's anxiety, depression and other negative emotions. With significant clinical nursing effect, it can improve patient's treatment compliance, thus results in speedy recovery.
\end{abstract}

Key words: Humanistic nursing care, chronic pelvic inflammatory disease, clinical effect, negative feelings, nursing satisfaction

Chronic pelvic inflammatory disease is relatively a common gynecological disease usually due to failure of timely and thorough treatment of acute pelvic inflammatory disease. Moreover, patients have poor constitution, so acute course of disease translates into chronic diseases, causing repeated attacks, delayed healing and eventually leading to chronic pelvic inflammatory disease ${ }^{[1]}$. Some patients have chronic pelvic inflammatory disease without acute pelvic inflammatory disease, for instance, suffering from salpingitis after infection of Chlamydia trachomatis ${ }^{[2]}$. The disease can be recurrent with long course. There is no obvious clinical manifestation, generally low fever, fatigue, lumbosacral pain, lower abdominal pain, painful intercourse, increased vaginal discharge, menstrual disorders, etc. Severe cases can cause infertility ${ }^{[3,4]}$. In clinical treatment process, treatment account for an important part. However, effectiveness and reasonableness of nursing method and nursing interventions also seriously affect patient's clinical treatment outcome ${ }^{[5-9]}$. The results of this study indicate that in clinical treatment course of patients with chronic pelvic inflammatory disease, implementation of humanistic nursing care interventions brings significant effect.

\section{MATERIALS AND METHODS}

Chronic pelvic inflammatory disease refers to chronic inflammation in female genital and pelvic peritoneum, surrounding connective tissues ${ }^{[10]}$. Pelvic inflammatory disease includes salpingitis, metritis, oophoritis, pelvic peritonitis and inflammation of connective tissue ${ }^{[11,12]}$. Female pelvic structure and uterine anatomical structure is shown in fig. 1 and fig. 2. Timely treatment of the disease plus effective and reasonable nursing measures is the key to improve patient's clinical efficacy and prognosis.

This research method is to apply humanistic nursing care and clinical care of patients with chronic pelvic inflammatory disease. Provide 45 patients with conventional treatment and care of chronic pelvic inflammatory disease, including antibiotic antiinfective therapy, medication care and guidance, continuous observation. Meanwhile, actively implement humanistic nursing care. Nursing case flow 


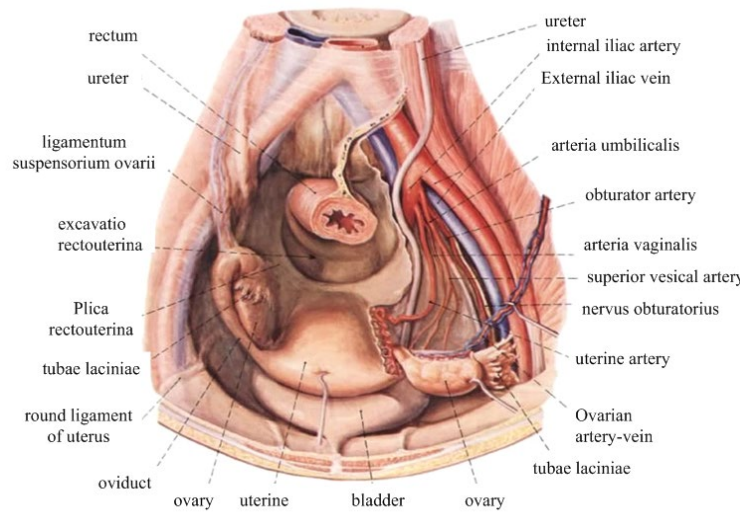

Fig. 1: Female pelvic structure diagram (anterosuperior view)

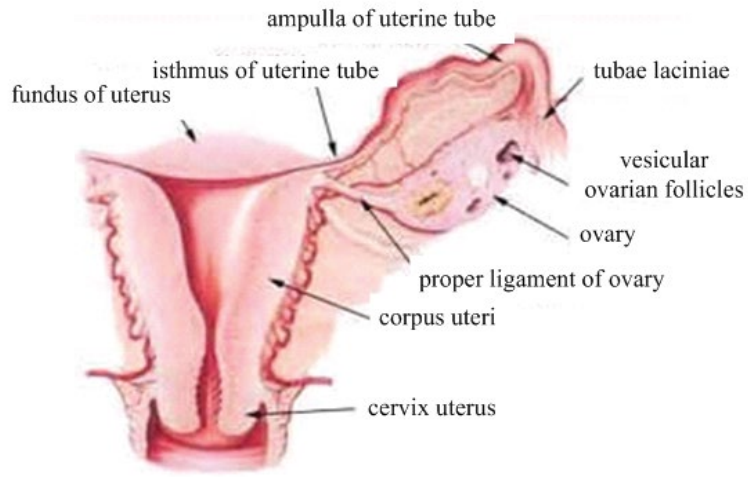

Fig. 2: Uterine coronal section

chart during patients hospitalization is shown in fig. 3, with specific intervention measures as follows.

After patients are admitted to hospital, actively make a self-introduction to patients, introduce ward environment and relevant staff to them, inform patients of related rules and regulations of hospitals and departments, provide patients with quiet and comfortable, airy and bright ward environment with suitable humidity, suitable temperature, place marked designation card, warning signs, etc. at bed side, regularly disinfect items on ground and air in the ward to avoid hospital infections ${ }^{[13]}$. Conduct disease knowledge education among patients, tell patients reasons for the attack of diseases, clinical manifestations, examination and treatment methods, prognosis, complications and related knowledge, so that patients grasp more knowledge of the disease, avoid excessive worry about the disease and treatment effect, depression, anxiety and other negative emotions, which causes impact on treatment compliance and efficacy.

During patient's hospitalization, provide psychological care intervention in the whole course. As the saying goes, "three points go to treatment, seven points go to care", patients psychological state exerts a significant impact on treatment effect and prognostic rehabilitation of the disease. Clinical study shows that in good mental state, patient's prognosis is significant. Therefore, in the process of care, nurses should observe patients closely, implement psychological care for patient's pessimism, depression, anxiety, provide targeted psychological care intervention in combination with patients conditions so that patients treatment compliance improve. Patients attention can be diverted through listening to music, reading, watching $\mathrm{TV}$, etc. so as to ease psychological pressure. Dietary reasonableness also significantly enhances patient's constitution and resistance, which can improve the body's ability to resist viruses and promote disease recovery. During personalized care, evaluate patients physical condition and state of illness, develop individualized rational, scientific diet program with high protein, high calorie, high vitamin diet as the main. Avoid spicy, cold and other irritable food, take food quantitatively on time. More intake of food containing crude fiber can facilitate enhancement of gastrointestinal motility. In the process of drug therapy, guide patients in medication, inform patients, name of the drug, pharmacological effect, adverse reactions that may occur after medication and actively provide treatment.

On the day patients are discharged, conduct discharge education among patients, inform them of relevant precautions after discharge and tell them to enhance

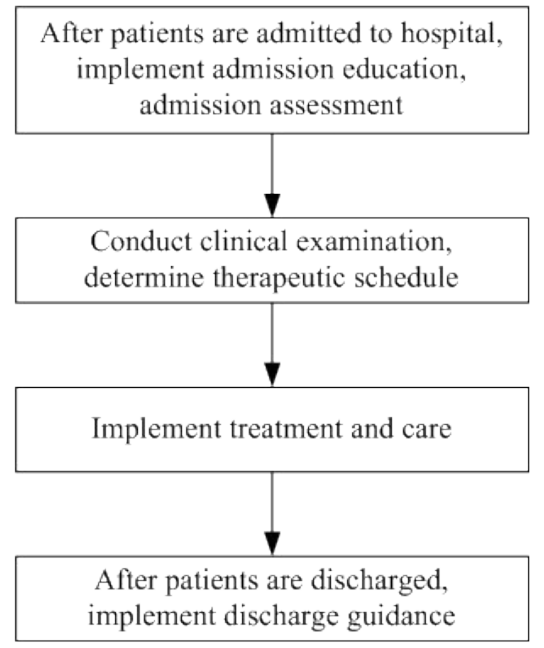

Fig. 3: Patient hospitalization care flow chart 
nutrition, proper exercises, timely hospitalization for treatment in case of discomfort, regularly go to hospital for review. Encourage and help patients to establish good habits to enhance body resistance.

After completing nursing intervention, compare patients psychological status change before and after care with self-rating anxiety scale (SAS), self-rating depression scale (SDS). SAS, SDS assessment grading criteria is shown in fig. 4 and fig. 5.

Assess patient's clinical nursing efficacy, whose evaluation standard includes cured, valid and invalid, particularly as shown in fig. 6 .

\section{RESULTS AND DISCUSSION}

Before nursing, patients SAS score was (73.58 \pm 4.36$)$ points which decreases to $(48.39 \pm 2.47)$ points after nursing. Before nursing, SDS score was $(75.86 \pm 4.28)$ points which decreases to $(46.63 \pm 3.72)$ points after

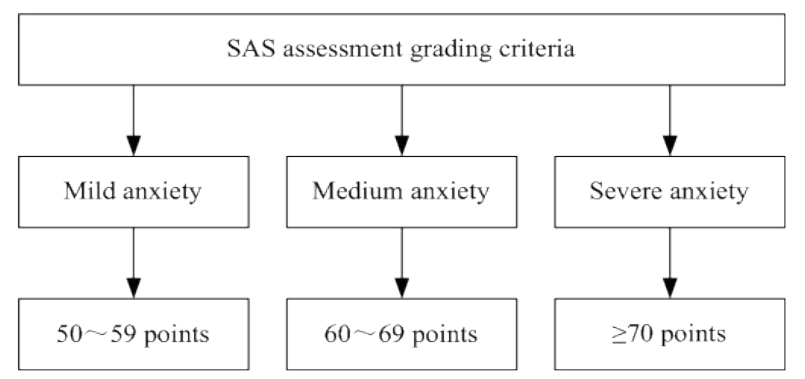

Fig. 4: SAS assessment grading criteria

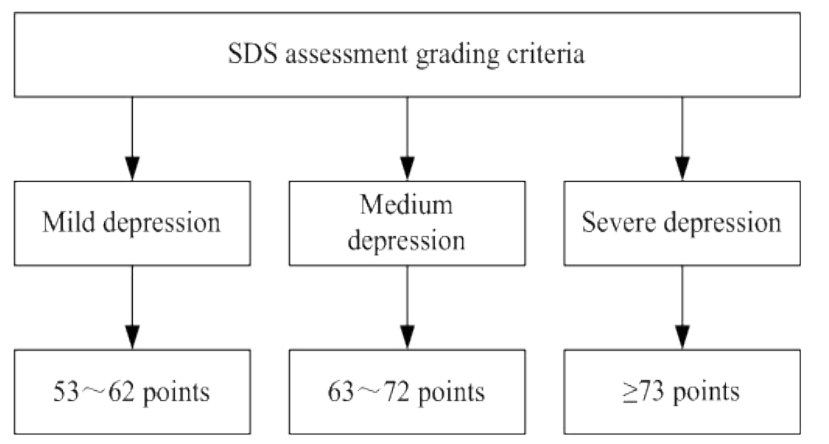

nursing. It can be seen that, after implementation of humanistic nursing care intervention, patient's anxiety, depression is significantly relieved, significantly lower than the scores before nursing, specifically shown in Table 1.

After this nursing, cases with marked, valid and invalid effect are 25, 16 and 4 respectively, accounting for $55.6 \%, 35.6 \%, 8.9 \%$. Total effective nursing rate is the sum of marked and valid rates that are total valid nursing cases are 41 , with total effective rate at $91.1 \%$.

Chronic pelvic inflammatory disease, a common gynecological disease, refers to chronic inflammatory diseases at internal genitalia and surrounding pelvic peritoneum, connective tissues. Ovarian cysts, hydrosalpinx, pelvic connective tissue inflammation, salpingitis, etc. are types of pelvic inflammatory disease. Currently, in China, incidence of the disease is high, mainly due to certain limitations in China's health and medical conditions ${ }^{[14]}$. After chronic pelvic inflammatory disease leads to chronic adnexitis, there will be palpable mass in check. Incidence of pelvic inflammation may also be caused by infection or spread of adjacent organs, for example, pelvic inflammation caused by external genitalia inflammation infection ${ }^{[15]}$. Chronic pelvic inflammatory disease can usually lead to bilateral oviduct inflammation, forming oviduct opening as time passes and causing partial interlock at the umbrella end or complete interlock. In addition, chronic pelvic inflammatory disease can also cause narrowing, adhesions or lumen atresia of mucous membrane within the oviduct ${ }^{[16]}$. Under normal circumstances, natural defense capacity of the female reproductive system is relatively strong, with a barrier effect for bacteria, pathogens, etc. Invasion occurs when natural defense capacity of the body declines, possibility of occurrence of chronic pelvic inflammatory disease significantly improves. Main pathogenic factors of chronic pelvic inflammatory disease include abortion, bad sexual habits, postoperative infection

Fig. 5: SDS assessment grading criteria

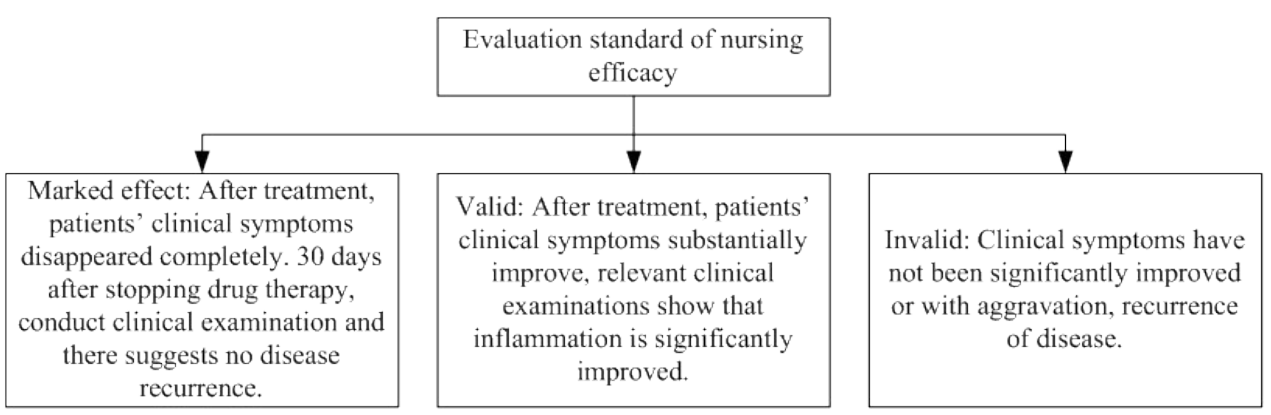

Fig. 6: Evaluation criteria for humanistic nursing care efficacy 
TABLE 1: PATIENTS ANXIETY AND DEPRESSION CHANGES BEFORE AND AFTER NURSING (POINT)

\begin{tabular}{lcc}
\hline Time & SAS score & SDS score \\
\hline Before nursing & $73.58 \pm 4.36$ & $75.86 \pm 4.28$ \\
After nursing & $48.39 \pm 2.47$ & $46.63 \pm 3.72$ \\
\hline
\end{tabular}

in uterine cavity, postpartum infection, adjacent organ inflammation diffusion, etc. ${ }^{[17]}$. In severe cases, inflammation may cause diffusive infection of uterus, pelvic peritoneum, adjacent organs of the uterus and can even lead to infertility. Therefore, early treatment of patients is the key to improve prognosis efficacy of patients and patients living quality.

Adult's rectum is with rich blood circulation and can absorb drug in passive diffusion form when administered with drug therapy. There are many clinical treatment methods for patients with chronic pelvic inflammatory disease, of which, rectal administration is one of common treatment methods. The treatment method enables drug to quickly and directly reach and act in the pelvic cavity. With relatively high drug concentration, it can effectively improve clinical treatment effect. Furthermore, rectal administration can reduce drug irritation on gastrointestinal tract, reducing enzyme, gastric acid and other substances in the gastrointestinal tract which affects drug efficacy ${ }^{[18]}$.

Humanistic nursing care is a significant care measure that improves patients clinical treatment effect through implementation of humanistic nursing care interventions on patient's psychology, physique ${ }^{[19,20]}$. After patients are admitted to hospital, faced with unfamiliar environment plus concerns about the disease, most patients will have negative emotions such as stress, anxiety, depression, fear. Therefore, to implement psychological care intervention after patient's admission, provide psychological care with combination of patient's specific circumstances and emotional characteristics and create a user friendly atmosphere, so that patients receive understanding, care and respect during hospitalization and that patient's treatment compliance improves help to improve treatment effect and living quality after intervention.

This research reveals that implementation of humanistic nursing care in clinical diagnosis and treatment of patients with chronic pelvic inflammatory disease can effectively relieve patient's negative emotions such as anxiety and depression. With relatively high nursing effect, it helps to improve patient's prognosis and living quality. Thus, humanistic nursing intervention is worthy to be popularized in clinical treatment of patients with chronic pelvic inflammatory disease.

\section{Acknowledgements:}

These authors contributed equally to this work.

\section{Conflict of Interests:}

The authors declared no conflict of interest.

\section{REFERENCES}

1. Feng X, Zhu C, Wu B. Analysis of clinical effect of applying whole-course nursing interventions in patients with chronic pelvic inflammatory disease (with 66 cases reported). Guide Chin Med 2013;20(7):344-5.

2. Ma J. Analysis of effect of holistic nursing care on 163 cases of patients with chronic pelvic inflammatory disease. Chin Foreign Med Treat 2013;18(13):134-5.

3. Chen L, Kang B. Analysis of humanistic nursing case for chronic pelvic inflammatory disease. Med Innov Chin 2013;30(21):69-71.

4. Lin H.Application of humanistic nursing care in comprehensive treatment of 23 cases of chronic pelvic inflammatory diseases. Chin J Ethnomed Ethnopharm 2014;26(6):79.

5. Wu J, Zhang L, Yin S, Wang H, Wang G, Yuan J. Differential diagnosis model of hypocellular myelodysplastic syndrome and aplastic anemia based on the medical big data platform. Complexity 2018:1-12.

6. Li W, Jia MX, Deng J, Wang JH, Lin QL, Liu C, et al. Isolation, genetic identification and degradation characteristics of CODdegrading bacterial strain in slaughter wastewater. Saudi J Biol Sci 2018;25(8):1800-5.

7. Ren Y, Jiao X, Zhang L. Expression level of fibroblast growth factor 5 (FGF5) in the peripheral blood of primary hypertension and its clinical significance. Saudi J Biol Sci 2018;25(3):46973.

8. Lou Y, Yang J, Wang L, Chen X, Xin X, Liu Y. The clinical efficacy study of treatment to Chiari malformation type I with syringomyelia under the minimally invasive surgery of resection of Submeningeal cerebellar Tonsillar Herniation and reconstruction of Cisterna magna. Saudi J Biol Sci 2019;26(8):1927-31.

9. Li W, Jia MX, Wang JH, Lu JL, Deng J, Tang JX, et al. Association of MMP9-1562C/T and MMP13-77A/G polymorphisms with non-small cell lung cancer in southern Chinese population. Biomolecules 2019;9(3):107.

10. He F, Liu Y, Chen S. Correlation analysis of chronic pelvic pain and anxiety plus nursing care. Chin Foreign Med Treat 2014;17(15):154-7.

11. Zheng W, Chen L. The roles of attentional shifts and attentional reengagement in resolving the spatial compatibility effect in tactile simon-like tasks. Sci Rep 2018;8(1):1-11.

12. Li H, Cao Z, Xu J, Wang F, Xiong R, Xu Z, et al. Cerebrospinal fluid FGF23 levels correlate with a measure of impulsivity. Psychiatry Res 2018;264:394-7.

13. Gonzalez AA, Grases F, Mari B, Tomas-Salva M, Rodriguez A. Urinary phytate concentration and risk of fracture determined by the FRAX index in a group of postmenopausal women. Turk J Med Sci 2019;49(2):458-63.

14. Zhao Q, Zhao C. Clinical efficacy analysis of improved retention enema for chronic pelvic inflammatory disease. Chin Mod Med 2014;26(21):67-9. 
15. Zhou Y, Liang J, Wei $\mathrm{H}$, Meifen M, Xiujin L. Effect observation and clinical nursing of treating 50 cases of chronic pelvic inflammatory diseases with TCM enema combined with physiotherapy. J Qilu Nurs 2015;43(1):73-5.

16. Wang Z. Analysis of applying nursing intervention in 27 cases of chronic pelvic inflammatory diseases. Chin J Ethnomed Ethnopharm 2015;38(4):129-31.

17. Aisha NM, Coulee Bahar CI. Clinical analysis of treating chronic pelvic inflammatory disease with integrative Chinese and western medicine and nursing care. Chin Health Ind 2012;35(18):52.

18. Chu X, Li S. Analysis of application value of psychological intervention in chronic pelvic inflammatory disease nursing. Chin Contin Med Educ 2015;30(20):216-7.

19. Jia Q, Li Z, Guo C, Huang X, Kang M, Song Y, et al. PEGMA- modified bimetallic NiCo Prussian blue analogue doped with Tb (III) ions: Efficiently pH-responsive and controlled release system for anticancer drug. Chem Eng J 2020;389:124468.

20. Pati NB, Gupta VR, Mayasa V, Velivela S, Hussain MA. Rethinking chronic pain treatment with opioids. Indian J Pharm Sci 2018;79(6):849-57.

This is an open access article distributed under the terms of the Creative Commons Attribution-NonCommercial-ShareAlike 3.0 License, which allows others to remix, tweak, and build upon the work non-commercially, as long as the author is credited and the new creations are licensed under the identical terms

This article was originally published in a special issue, "Trends in Therapeutic Management of Various Clinical Conditions II" Indian J Pharm Sci 2021:83(2)Spl issue;114-118 